\title{
DeltaNp63 expression is a biomarker to predict survival after recurrence of upper urinary tract urothelial carcinoma
}

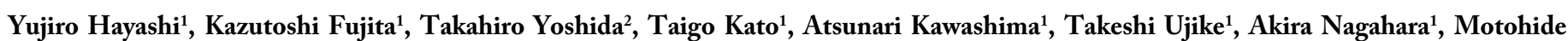
Uemura $^{1,3}$, Masahiro Inoue ${ }^{4}$, Hiroaki Fushimi ${ }^{5}$, Ryoichi Imamura ${ }^{1}$, Seiji Yamaguchi ${ }^{6}$, Hiroshi Miyamoto ${ }^{7}$ and Norio Nonomura ${ }^{1}$

${ }^{1}$ Department of Urology, Osaka University Graduate School of Medicine, Japan

${ }^{2}$ The James Buchanan Brady Urological Institute and Department of Urology, Johns Hopkins School of Medicine, USA

${ }^{3}$ Department of Therapeutic Urologic Oncology, Osaka University Graduate School of Medicine, Japan

${ }^{4}$ Department of Biochemistry, Osaka International Cancer Center, Japan

${ }^{5}$ Department of Pathology, Osaka General Medical Center, Japan

${ }^{6}$ Department of Urology, Osaka General Medical Center, Japan

${ }^{7}$ Departments of Pathology \& Laboratory Medicine, Urology, and Oncology, University of Rochester Medical Center, USA

\begin{abstract}
TP63 is a key regulator of epithelial development and homeostasis, but its role in cancer progression remains unclear. In this study, we assessed the usefulness of $\triangle \mathrm{Np} 63$ (predominant isoform of TP63) as a prognostic biomarker of upper tract urothelial carcinoma (UTUC) that is a relatively uncommon cancer and is often associated with poor outcome.

We investigated the immunoreactivity of $\Delta \mathrm{Np} 63$ in radical nephroureterectomy specimens on tissue microarrays containing samples from 83 patients with UTUC There were no significant associations between $\Delta \mathrm{Np} 63$ expression and tumor grade/stage, disease progression, or cancer-specific survival (CSS). However, in subgroup analysis of 32 patients who experienced disease recurrence after radical nephroureterectomy and subsequently received platinum-based chemotherapy showed that high $\Delta \mathrm{Np} 63$ expression was associated with better CSS $(\mathrm{P}<0.05)$. Our study indicated that $\Delta \mathrm{Np} 63$ expression could be a significant prognostic biomarker and a promising factor for predicting chemo-sensitivity in patients with UTUC.
\end{abstract}

\section{Introduction}

Upper tract urothelial carcinoma (UTUC) is a relatively rare malignancy and accounts for only a small number of urothelial carcinoma [1-3]. Because patients with UTUC often exhibit only mild symptoms, it is difficult to diagnose at the early stage. Indeed, about $60 \%$ of UTUCs are invasive at diagnosis compared with 15$25 \%$ of bladder tumors $[4,5]$. Radical nephroureterectomy is offered as a standard treatment for localized tumors. Systemic chemotherapy is administered for patients who have metastasis at diagnosis or experience recurrence after surgery, but the prognosis is poor in most cases [6]. Although pathological stage and tumor grade are associated with tumor progression and poor survival $[7,8]$, it is difficult for physicians guided only by these histopathological factors to predict prognosis and decide whether to offer adjuvant chemotherapy in a precise manner for individual UTUC patients. A greater understanding of the biological behavior of tumors is necessary for the realization of precision medicine and improvement of patient outcomes.

TP63 is a member of the TP53 family, and this gene is composed of 15 exons, spanning over $270,000 \mathrm{bp}$ on chromosome $3 \mathrm{q} 27[9,10]$. The Tp63 gene has two transcriptional start sites: one contains an $\mathrm{N}$-terminal transactivation (TA) domain (TAp63), and the others don't $(\triangle \mathrm{Np} 63)$. Both genes can be alternatively spliced to generate proteins with three types of C-termini ( $\alpha, \beta$, and $\gamma$ ). TP63 is constitutively expressed in the nuclei of epithelial cells and acts as a key regulator of development and homeostasis of epithelium, but the role of TP63 in cancer progression has not been fully understood. In human urothelial tissues and urothelial carcinomas, $\Delta \mathrm{Np} 63$ is predominantly expressed compared to TAp63 [11-13]. Loss of $\Delta \mathrm{Np} 63$ expression is associated with more advanced disease and acquisition of epithelial-mesenchymal transition[13-16], while it's expression in muscle-invasive bladder cancer correlates with a worse prognosis [11,17]. Fukushima et al demonstrated loss of $\Delta \mathrm{Np} 63$ expression during muscle-invasive recurrence progressed from low-grade papillary noninvasive bladder tumor, accompanied by $\mathrm{N}$-cadherin up-regulation, indicating that $\Delta \mathrm{Np} 63$ could suppress invasion of urothelial bladder carcinoma cells [18].

Thus TP63 likely plays a complex role in tumor formation and progression of urothelial cancer. In this study, we investigate the prognostic significance of $\Delta \mathrm{Np} 63$ expression in UTUC patients.

\section{Material and methods}

\section{Patients and tissue samples}

A tissue micro-array (TMA) of UTUC specimens was constructed with spotted triplicate tumor samples from 83 patients who underwent

Correspondence to: Kazutoshi Fujita, Department of Urology, Osaka University Graduate School of Medicine, 2-2 Yamadaoka, Suita, Osaka 565-0871, Japan, Tel: +81-6-6879-3531, Fax: +81-6-6879-3539, E-mail: kazufujita2@gmail.com

Received: February 02, 2018; Accepted: February 21, 2018; Published: February 24,2018 
radical nephroureterectomy performed with curative intent between 1999 and 2011 at Osaka General Medical Center, Osaka, Japan. Appropriate approval was obtained from the local institutional review board before construction and use of the TMA, and written informed consent was obtained from all patients. Clinicopathological characteristics of the patients were obtained from medical records and follow-up data. Tumor progression was defined as the development of recurrence at the site of radical nephroureterectomy, lymph node metastasis, and/or visceral metastasis. Metachronous or synchronous lower tract recurrence (e.g., in the bladder) was not defined as tumor progression. Patients were followed up from initial diagnosis to the appearance of the event of interest or the end of the study. Patients who did not present the event of interest by the end of the study were censored from time-to-event analyses.

\section{Immunohistochemistry}

Immunohistochemical staining was performed on $5-\mu \mathrm{m}$ sections from the UTUC TMA. Sections were deparaffinized, rehydrated, and subjected to heat-induced antigen retrieval in $10 \mathrm{mM}$ citrate buffer $(\mathrm{pH}$ 6.0) at $121^{\circ} \mathrm{C}$ for $15 \mathrm{~min}$ before staining, and endogenous peroxidase activity was quenched with $\mathrm{H}_{2} \mathrm{O}_{2}$. Sections were then incubated with a primary antibody against $\Delta \mathrm{Np} 63$ (p40, Calbiochem, Merck Millipore, Darmstadt, Germany). After incubation with a biotinylated antibody and treatment with the Vectastain $\mathrm{ABC}$ Kit (Vector Laboratories, Burlingame, Burlingame, USA), peroxidase activity was visualized with Vector NovaRED (Vector Laboratories, Burlingame, USA) according to the manufacturer's instructions. The sections were counterstained with hematoxylin. All of the stained sections were manually scored by researchers who were blinded to sample identity.

\section{Scoring system}

Nuclear staining of $\Delta \mathrm{Np} 63$ was evaluated for both intensity and extent (percentage of positive cells). An "H score" was assigned to each TMA spot as the sum of the products of the intensity ( 0 , negative; 1 , weakly positive; 2 , moderately positive; and 3 , strongly positive) and the extent of immunoexpression (0 to $100 \%$ ), obtaining a value from 0 to 300 , as previously described [19]. The final $\mathrm{H}$ score for each case was defined as the average score of triplicate TMA spots and was used during statistical analyses. For statistical analysis, the patients were divided into two groups according to the $\mathrm{H}$ score (High group: $\mathrm{H}$ score $>$ the median, and Low group: H score $\leq$ the median).

\section{Statistical analysis}

Statistical analyses were performed using JMP ${ }^{\circledR}$ Pro 13.2.0 (SAS Institute Inc., Cary, NC). Patient characteristics were compared using the Mann-Whitney $U$ test and $\chi^{2}$-test. The survival rates were determined using the Kaplan-Meier method and compared with the log-rank test.

\section{Results}

Table 1 shows the characteristics of the 83 patients. This cohort consisted of 47 male and 36 female patients, 48 to 87 years of age (median: 71 years), with low-grade urothelial carcinoma (in 11 patients) and high-grade urothelial carcinoma (in 72 patients). The carcinomas corresponded to non-muscle-invasive tumors ( $\mathrm{pTa}$ or pT1) in 31 patients and muscle-invasive tumors (pT2, pT3, or pT4) in 52 patients. Pathological lymph node metastasis $(\mathrm{pN}+)$ was noted in 11 patients. The follow up period (measured from the date of radical nephroureterectomy to the time of last follow-up visit) ranged from 2 to 139 months. The median cancer specific survival (CSS) time was
Table 1. Histopathological features of patients with upper tract urothelial carcinoma

\begin{tabular}{|c|c|c|c|}
\hline Feature & $\mathbf{\Delta N p 6 3} \mathbf{h i g h}$ & $\mathbf{\Delta N p 6 3}$ low & Total \\
\hline Patients & 43 & 40 & 83 \\
\hline Sex (male/female) & $27 / 16$ & $20 / 20$ & $47 / 36$ \\
\hline $\begin{array}{c}\text { Median age, yrs } \\
\text { (range) }\end{array}$ & $71(48-87)$ & $71(53-85)$ & $71(48-87)$ \\
\hline Histological grade & & & \\
\hline G1 & $5(11.6 \%)$ & $1(2.5 \%)$ & $6(7.2 \%)$ \\
\hline G2 & $18(41.8 \%)$ & $15(37.5 \%)$ & $33(39.7 \%)$ \\
\hline G3 & $20(46.5 \%)$ & $24(60.0 \%)$ & $44(53 \%)$ \\
\hline Pathological stage & & & $19(22.8 \%)$ \\
\hline pTa & $8(18.6 \%)$ & $11(27.5 \%)$ & $12(14.4 \%)$ \\
\hline pT1 & $7(16.2 \%)$ & $5(12.5 \%)$ & $7(8.4 \%)$ \\
\hline pT2 & $5(11.6 \%)$ & $2(5.0 \%)$ & $39(46.9 \%)$ \\
\hline pT3 & $21(48.8 \%)$ & $18(45.0 \%)$ & $6(7.2 \%)$ \\
\hline pT4 & $2(4.6 \%)$ & $4(10.0 \%)$ & $11(13.2 \%)$ \\
\hline pN+ & $5(11.6 \%)$ & $6(15.0 \%)$ & $31(37.3 \%)$ \\
\hline LVI & & & $52(62.6 \%)$ \\
\hline present & $16(37.2 \%)$ & $15(37.5 \%)$ & \\
\hline absent & $27(62.7 \%)$ & $25(62.5 \%)$ & \\
\hline
\end{tabular}

LVI: Lymphovascular Invasion

46 months. During this period, 34 patients (41.0\%) experienced local or distant tumor recurrence, and $22(26.5 \%)$ experienced bladder recurrence (Figure 1).

Representative patterns of immunoexpression are depicted in Figure 1. $\Delta$ Np63 staining was strong in 22 cases $(26.5 \%)$, moderate in $33(39.8 \%)$, weak in $17(20.5 \%)$ and absent in $11(13.2 \%) . \Delta \mathrm{Np} 63$ immunoreactivity was not associated with pathological stage or tumor grade. To evaluate the prognostic values of $\Delta \mathrm{Np} 63$ expression in UTUC, we performed Kaplan-Meier analysis coupled with the logrank test. $\triangle \mathrm{Np} 63$ expression was not associated with cancer-specific mortality or disease recurrence in lymph node or distant metastasis in overall cohort (Figures 2A,2B). However, in subgroup analysis of 32 patients (including 8 patients who underwent adjuvant platinumbased chemotherapy) who experienced the disease recurrence after radical nephroureterectomy and subsequently received platinumbased chemotherapy, high $\Delta \mathrm{Np} 63$ expression was associated with better CSS (Figure 2C).

\section{Discussion}

In this study, we showed the expression of $\Delta$ Np63 in radical nephroureterectomy specimens was significantly correlated with CSS in patients who experienced recurrence after surgery and received platinum-based chemotherapy, while the expression of $\Delta \mathrm{Np} 63$ has no association with cancer-specific mortality or disease recurrence in lymph node or distant metastasis in overall cohort.

However, there have been many studies demonstrating the association of $\Delta \mathrm{Np} 63$ expression with disease aggressiveness and prognosis of urothelial bladder cancer [11-18], while the expression of $\Delta \mathrm{Np63}$ shows no significant association with cancer-specific mortality in our UTUC cohort. This is possibly due to differences in treatment strategy (e.g., lymphadenectomy, perioperative chemotherapy), embryology and cancer biology between upper tract and bladder tumor. Further studies are needed to clarify this discrepancy.

The expression of $\Delta \mathrm{Np} 63$ in radical nephroureterectomy specimens showed significant correlation with CSS of patients who experienced recurrence after surgery and subsequently received platinum-based chemotherapy. In muscle invasive bladder cancer, Choi et al reported that "basal tumors" characterized by p63 activation has good response 

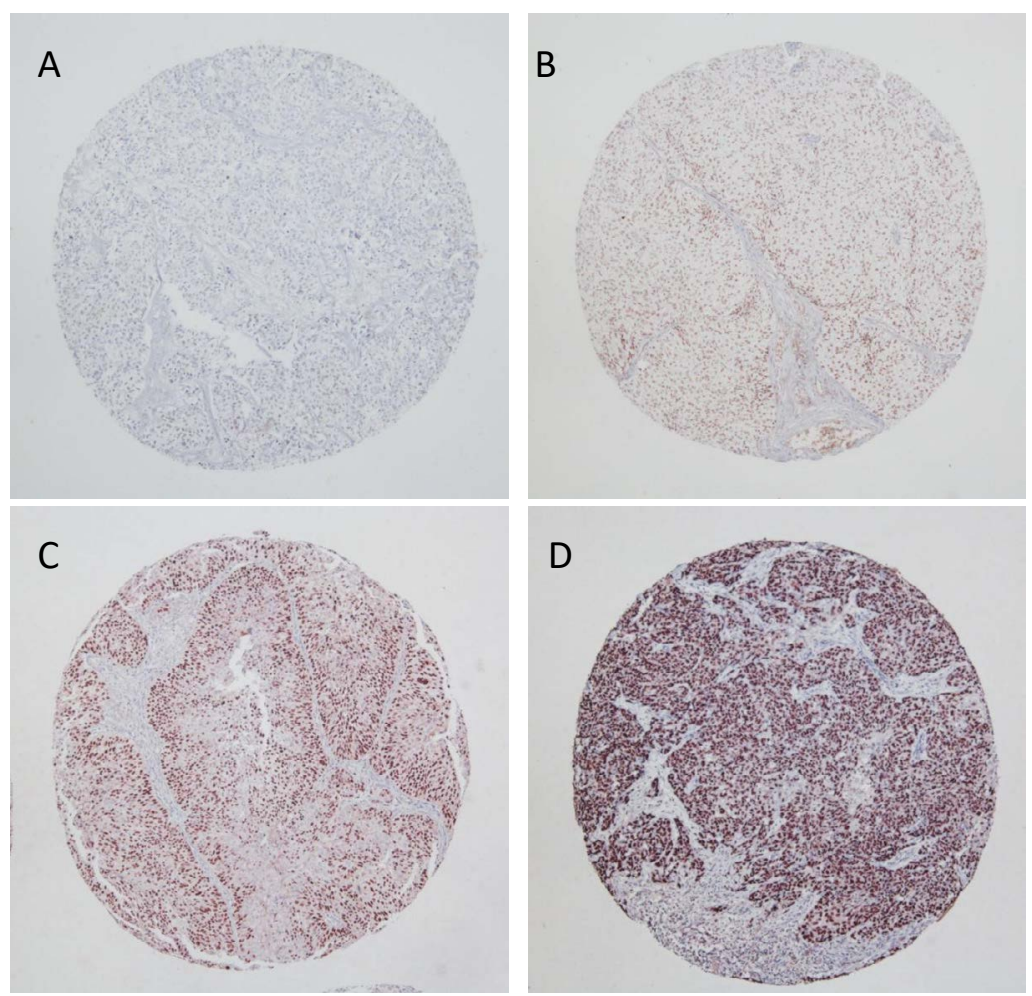

Figure 1. Typical patterns of immunohistochemical expression of $\Delta \mathrm{Np} 63$ (A: negative, B: weakly positive, C: moderately positive, D: strongly positive) in upper tract urothelial carcinoma tissues. Original magnification: $\times 200$.
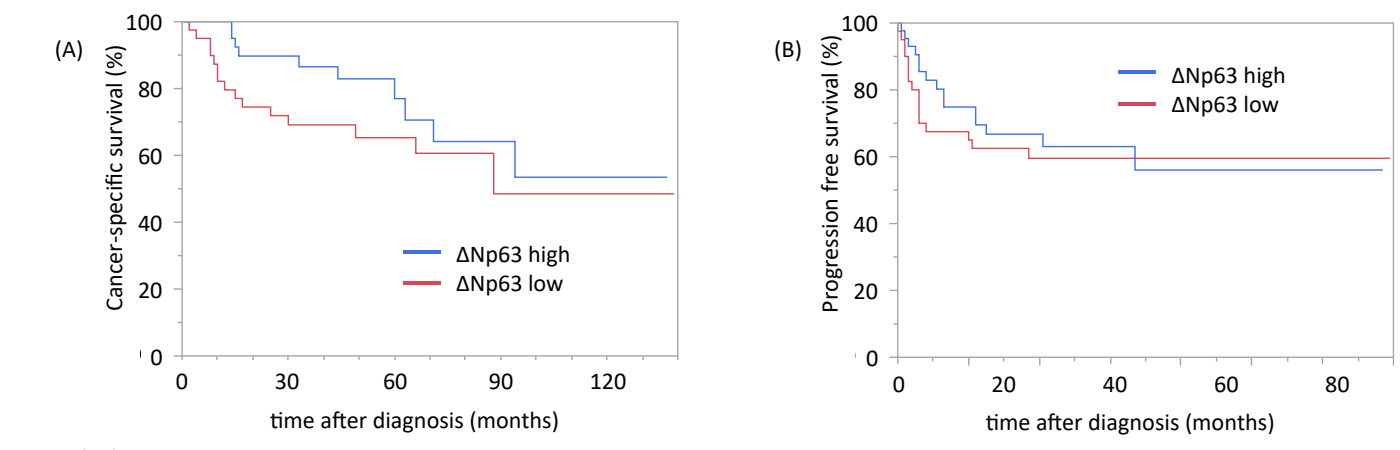

$\begin{array}{llllll}\Delta \text { Np63 high } & 43 & 28 & 12 & 6 & 2 \\ \Delta \text { Np63 low } & 40 & 25 & 14 & 3 & 1\end{array}$

$\begin{array}{llllll}\Delta \text { Np63 high } & 43 & 28 & 18 & 9 & 7 \\ \Delta \text { Np63 low } & 40 & 26 & 19 & 11 & 8\end{array}$

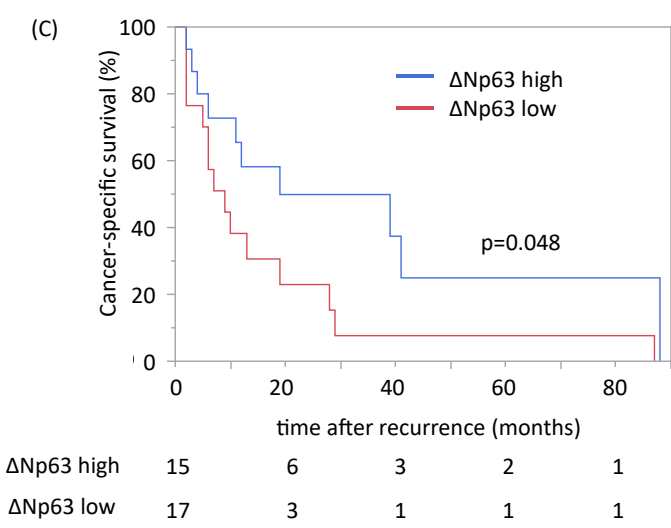

Figure 2. Cancer-specific survival rate and progression-free recurrence survival rate in patients with UTUC according to the expression levels of $\Delta$ Np63 (A: cancer-specific survival of overall cohort, B: progression-free survival of overall cohort, C: cancer-specific survival of patients who experienced recurrence after nephroureterectomy and received platinum-based chemotherapy). 
to neoadjuvant chemotherapy [20]. $\Delta \mathrm{Np} 63$ could thus be a useful marker for predicting chemo-sensitivity in UTUC patients who have recurrence or metastasis after radical nephroureterectomy.

This study has some limitations. First, we used TMA samples instead of whole tumor sections and the heterogeneity of staining could affect the evaluation of the expression levels. However, several studies have shown that multiple TMA spots adequately represent the expression of an entire section in the assessment of immunohistochemical markers [21]. Second, this retrospective data lacks clinical information (e.g., blood test findings, performance status, and detail information of chemotherapy), it was difficult to investigate the association of chemosensitivity with $\triangle \mathrm{Np} 63$ expression in patients with UTUC thoroughly.

\section{Conclusion}

We demonstrated that $\Delta \mathrm{Np} 63$ expression immunohistochemically determined in a set of TMA consisting of radical nephroureterectomy specimens could be a novel biomarker for CSS and response to chemotherapy in patients who had recurrence after surgery.

\section{Data availability}

The datasets analyzed during the current study are available from the corresponding author on reasonable request.

\section{References}

1. Kim HS, Jeong CW, Kwak C, Kim HH, Ku JH (2017) Association between demographic factors and prognosis in urothelial carcinoma of the upper urinary tract: a systematic review and meta-analysis. Oncotarget 31: 7464-7476. [Crossref]

2. Miyazaki J, Nishiyama H1 (2017) Epidemiology of urothelial carcinoma. Int J Urol 24: 730-734. [Crossref]

3. Raman JD, Messer J, Sielatycki JA, Hollenbeak CS (2011) Incidence and survival of patients with carcinoma of the ureter and renal pelvis in the USA, 1973-2005. BJU Int 107: 1059-1056. [Crossref]

4. Babjuk M, Oosterlinck W, Sylvester R, Kaasinen E, Böhle A, et al. (2011) EAU guidelines on non-muscle-invasive urothelial carcinoma of the bladder, the 2011 update. Eur Urol 2011; 59: 997-1008. [Crossref]

5. Margulis V, Shariat SF, Matin SF, Kamat AM, Zigeuner R, et al. (2009) Upper Tract Urothelial Carcinoma Collaboration. Outcomes of radical nephroureterectomy: a series from the Upper Tract Urothelial Carcinoma Collaboration. Cancer 115: 1224-1233. [Crossref]

6. Seisen T, Krasnow RE, Bellmunt J, Rouprêt M, Leow JJ, et al. (2017) Effectiveness of Adjuvant Chemotherapy After Radical Nephroureterectomy for Locally Advanced and or Positive Regional Lymph Node Upper Tract Urothelial Carcinoma. J Clin Oncol 35: 852-860. [Crossref]
7. Huben RP, Mounzer AM, Murphy GP (1988) Tumor grade and stage as prognostic variables in upper tract urothelial tumors. Cancer 62: 2016-2020. [Crossref]

8. Leitner CV, Ederer IA, de Martino M, Hofbauer SL, Lucca I, et al. (2016) Dynamic prognostication using conditional recurrence and progression estimates for patients with nonmuscle invasive bladder cancer. J Urol 196: 46-51. [Crossref]

9. McKeon F (2004) p63 and the epithelial stem cell: more than status quo? Genes Dev 18: 465-469. [Crossref]

10. Murray-Zmijewski F, Lane DP, Bourdon JC (2006) p53/p63/p73 isoforms: an orchestra of isoforms to harmonise cell differentiation and response to stress. Cell Death Differ 13: 962-972. [Crossref]

11. Choi W, Shah JB, Tran M, Svatek R, Marquis L, et al. (2012) p63 expression defines a lethal subset of muscle-invasive bladder cancers. PLoS One 7: e30206. [Crossref]

12. Tran MN, Choi W, Wszolek MF, Navai N, Lee IL, et al. (2013) The p63 protein isoform? Np63a inhibits epithelial-mesenchymal transition in human bladder cancer cells: role of MIR-205. J Biol Chem 288: 3275-3288. [Crossref]

13. Koga F, Kawakami S, Kumagai J, Takizawa T, Ando N, et al. (2003) Impaired Delta Np63 expression associates with reduced beta-catenin and aggressive phenotypes of urothelial neoplasms. Br J Cancer 88: 740-747. [Crossref]

14. Koga F, Kawakami S, Fujii Y, Saito K, Ohtsuka Y, et al. (2003) Impaired p63 expression associates with poor prognosis and uroplakin III expression in invasive urothelial carcinoma of the bladder. Clin Cancer Res 9: 5501-5507. [Crossref]

15. Zigeuner R, Tsybrovskyy O, Ratschek M, Rehak P, Lipsky K, et al. (2004) Prognostic impact of p63 and p53 expression in upper urinary tract transitional cell carcinoma. Urology 63: 1079-1083. [Crossref]

16. Urist MJ, Di Como CJ, Lu ML, Charytonowicz E, Verbel D, et al. (2002) Loss of p63 expression is associated with tumor progression in bladder cancer. Am J Pathol 161: 1199-1206. [Crossref]

17. Karni-Schmidt O, Castillo-Martin M, Shen TH, Gladoun N, Domingo-Domenech J, et al. (2011) Distinct expression profiles of p63 variants during urothelial development and bladder cancer progression. Am J Pathol 178: 1350-1360. [Crossref]

18. Fukushima H, Koga F, Kawakami S, Fujii Y, Yoshida S, et al. (2009) Loss of DeltaNp63alpha promotes invasion of urothelial carcinomas via N-cadherin/Src homology and collagen/extracellular signal-regulated kinase pathway. Cancer Res 69: 9263-9270. [Crossref]

19. Munari E, Fujita K, Faraj S, Chaux A, Gonzalez-Roibon N, et al. (2013) Dysregulation of mammalian target of rapamycin pathway in upper tract urothelial carcinoma. Hum Pathol 44: 2668-2676. [Crossref]

20. Choi W, Porten S, Kim S, Willis D, Plimack ER, et al. (2014) Identification of distinct basal and luminal subtypes of muscle-invasive bladder cancer with different sensitivities to frontline chemotherapy. Cancer Cell 25: 152-165. [Crossref]

21. Camp RL, Neumeister V, Rimm DL (2008) A decade of tissue microarrays: progress in the discovery and validation of cancer biomarkers. J Clin Oncol 26: 5630-5637. [Crossref]

Copyright: (C2018 Hayashi Y. This is an open-access article distributed under the terms of the Creative Commons Attribution License, which permits unrestricted use, distribution, and reproduction in any medium, provided the original author and source are credited. 